\title{
Biología reproductiva de Convolvulus chilensis (Convolvulaceae) en una población de Aucó (centro-norte de Chile)
}

\author{
Reproductive biology of Convolvulus chilensis (Convolvulaceae) in a population \\ of Aucó (north-central Chile)
}

\author{
LORENA H. SUÁREZ1 ${ }^{1}$, WILFREDO L. GONZÁLEZ² \& ERNESTO GIANOLI ${ }^{1 . *}$ \\ ${ }^{1}$ ECOBIOSIS, Departamento de Botánica, Universidad de Concepción, Casilla 160-C, Concepción, Chile \\ ${ }^{2}$ Departamento de Ciencias Ecológicas, Facultad de Ciencias, Universidad de Chile \\ *Autor para correspondencia; e-mail: egianoli@udec.cl
}

\begin{abstract}
RESUMEN
Convolvulus chilensis es una hierba perenne, única representante endémica de la familia Convolvulaceae en Chile. Se estudió el sistema de reproducción, fenología, morfología y longevidad floral de C. chilensis en una población natural ubicada en la localidad de Aucó, dentro de la Reserva Nacional Las Chinchillas, IV Región, Chile. Se montó un experimento de polinización controlada considerando los tratamientos de polinización natural, polinización cruzada, autopolinización manual, autopolinización automática y apomixis, evaluándose su efecto sobre la formación de frutos y el número de semillas producidas por fruto. Adicionalmente, se compararon los siguientes atributos de la progenie según tipo de polinización (autopolinización o polinización cruzada): peso de semilla, germinación, altura y número de hojas de plántulas de ocho semanas en condiciones de invernadero. Se encontró que C. chilensis es una especie autocompatible, parcialmente autógama (capaz de autopolinizarse sin mediador) y parcialmente apomíctica (capaz de producir semillas sin participación de gameto masculino). La longevidad floral fue estimada en 5,25 h. Durante este período, aproximadamente en $1,5 \mathrm{~h}$ hay disponibilidad de polen en los estambres. El período de floración se extiende por 22 semanas (agosto a enero). El tratamiento de apomixis presentó el menor porcentaje de formación de frutos y la menor cantidad de semillas por flor en comparación a los tratamientos de polinización natural, cruzada manual, autopolinización automática y autopolinización manual, los cuales no mostraron diferencias entre sí en ambos atributos. El tipo de polinización (autopolinización o polinización cruzada) no afecta el desempeño de la progenie en los atributos de semilla y plántula evaluados.
\end{abstract}

Palabras clave: Convolvulaceae, Convolvulus chilensis, sistema de reproducción, autocompatibilidad, autogamia.

\begin{abstract}
The perennial herb Convolvulus chilensis is the only endemic species of the Convolvulaceae in Chile. The breeding system, phenology, morphology and floral longevity of $C$. chilensis were studied in a natural population located at Aucó, Reserva Nacional Las Chinchillas, IV Región, Chile. A controlled pollination experiment was carried out considering the following treatments: natural pollination, cross-pollination, manual self-pollination, automatic self-pollination and apomixis, evaluating their effect on fruit formation and seed number per fruit. In addition, we evaluated the following traits of the progeny according to pollination type (selfing or outcrossing): seed weight, germination and seedling height and number of leaves after eight weeks of germination under green house conditions. We found Convolvulus chilensis is self-compatible, partially autogamous (capable of selfing without a pollinator agent) and partially apomictic (capable of producing seeds without male gamet). The floral longevity was estimated in $5.25 \mathrm{~h}$ During this period, the pollen on the stamens is available only during $1.5 \mathrm{~h}$. The blooming period lasted for 22 weeks (August to January). The apomixis treatment showed a lower fruit set and seed number per flower than natural, cross and self-pollination treatments, which did not show any difference between them in both traits. Pollination type (selfing or outcrossing) did not affect progeny performance in terms of the evaluated seed and seedling traits.
\end{abstract}

Key words: Convolvulaceae, Convolvulus chilensis, breeding system, self-compatibility, autogamy. 


\section{INTRODUCCIÓN}

Los sistemas de reproducción en las plantas con flor (Angiospermas) conforman un rango continuo que abarca desde la autogamia obligada (e.g., flores cleistógamas) hasta la polinización cruzada obligada (Barrett \& Harder 1996). Los sistemas reproductivos que presentan las especies, se relacionan con factores intrínsecos (filogenia, rasgos de historia de vida) y extrínse$\cos$ (ambiente, interacciones bióticas) (Lovett-Doust \& Lovett-Doust 1988). Los niveles particulares de reproducción cruzada de una especie resultan de la interacción entre las condiciones ambientales locales y las características demográficas, de historia de vida y genéticas de las poblaciones (Barrett \& Eckert 1990).

La relación existente entre el sistema de reproducción y la forma de vida de las plantas ha sido ampliamente estudiada (Stebbins 1950, Barrett et al. 1996, Morgan et al. 1997, Aarssen 2000). Algunos estudios comparativos muestran que las especies anuales presentan con mayor frecuencia sistemas autocompatibles que las especies perennes (Stebbins 1957, Martin 1970, Arroyo \& Squeo 1990, Arroyo \& Uslar 1993). La ocurrencia de autopolinización en especies anuales podría asegurar la reproducción cuando la polinización cruzada se ve desfavorecida por causa de escasez o ausencia de polinizadores, o de potenciales donantes de polen co-específicos ("reproductive assurance hypothesis": Stebbins 1950, pero véase Schoen et al. 1996 y Morgan et al. 1997). Por su parte, la mayor longevidad de las especies perennes les otorga más posibilidades de reproducción, mostrando con mayor frecuencia polinización cruzada (comúnmente asociada a mecanismos de autoincompatibilidad) y sistemas mixtos. Sin embargo, existen dos mecanismos que se oponen a la evolución de la autopolinización. El más estudiado es la depresión por endogamia (Lloyd 1979, Charlesworth \& Charlesworth 1987, Lloyd et al. 1992, Husband \& Schemske 1996), fenómeno que consiste en la reducción de la adecuación biológica de progenies endogámicas en relación con aquellas provenientes de polinización cruzada. Otro mecanismo, conocido como pollen discounting, se refiere al costo de utilizar para autopolinización polen que potencialmente podría destinarse a polinización cruzada (Chang \& Rausher 1998, Holsinger 2000).
Otros factores intrínsecos, tales como diseño y longevidad floral o tasas diarias de floración, pueden afectar la evolución del sistema de reproducción de una especie, a través de la incidencia directa o indirecta sobre la disponibilidad y comportamiento de los polinizadores (Barrett \& Harder 1996).

En la familia Convolvulaceae se ha estudiado los sistemas reproductivos de especies en los géneros Calystegia, Convolvulus, Evolvulus, Ipomoea, Jacquemontia y Merremia (Martin 1970, Schlising 1970, Stucky \& Beckmann 1982, Weaver \& Riley 1982, Murcia 1990, Devall \& Thien 1992, Willmontt \& Búrquez 1996, Ushimaru \& Kikuzawa 1999, Koptur 2001', Galetto et al. 2002, Pinto-Torres \& Koptur $2003^{2}$ ). En estos estudios se reportan casos de especies totalmente autoincompatibles, muy común en el genero Ipomoea, y casos de sistemas mixtos. Agrupando las especies consideradas en estos estudios según su forma de vida, se encuentra que las anuales presentan autocompatibilidad en un $100 \%$ de los casos, mientras que solo un $21 \%$ en las perennes-herbáceas. Este patrón fue sugerido previamente por Martín (1970), quien consideró mayoritariamente especies de Ipomoea.

Existen 1.500 especies de convolvuláceas en el mundo (Cronquist 1981), de las cuales solo 15 se encuentran en Chile (O'Donell 1957). El género Convolvulus, siendo el segundo más numeroso de la familia con 250 especies (Cronquist 1981), está representado en Chile por seis especies, de las cuales cinco son nativas. La hierba perenne Convolvulus chilensis Pers. es la única especie endémica de las seis representantes de su género en Chile. Es típica de hábitat costeros del norte y centro del país (O’Donell 1957) y aunque posee una amplia distribución latitudinal, las poblaciones no son localmente abundantes. En poblaciones del centro-norte de Chile no se ha observado reproducción clonal (E. Gianoli, resultados no publicados), lo que sugiere que su único modo de

\footnotetext{
KOPTUR S (2001) The floral biology and breeding system of Jacquemontia curtissii, an endemic morning glory of south Florida pine rocklands. Abstract Book of the Annual Meeting of Botanical Society of America at Albuquerque, New Mexico, USA. Abstract No 156: p. 38. 2 PINTO-TORRES E \& S KOPTUR (2003) Investigating the breeding system of an endangered coastal dune plant, Jacquemontia reclinata (Convolvulaceae). Abstract Book of the Annual Meeting of Botanical Society of America at Mobile, Alabama, USA. Abstract $\mathrm{N}^{\circ}$ 142: p. 141.
} 
propagación en condiciones naturales es a través de semillas. Particularmente en la población de estudio (Aucó, IV Región, Chile), la densidad poblacional es baja: 11.48 individuos $\mathrm{ha}^{-1}$ y se ha observado altos niveles de herbivoría por pequeños mamíferos: 58,93 $\pm 4,99 \%$ (media $\pm \mathrm{EE}$ ) de tallos ramoneados con respecto al total de tallos del individuo (E. Gianoli, resultados no publicados).

Dados los antecedentes recopilados para la familia, sería esperable que $C$. chilensis presentara un sistema de reproducción en el que dominara la reproducción cruzada, pues se trata de una especie perenne. El objetivo de este trabajo es determinar el sistema de reproducción de $C$. chilensis en condiciones naturales y evaluar el efecto del tipo de polinización (autopolinización o polinización cruzada) sobre los atributos y desempeño de la progenie, a fin de detectar la posible ocurrencia de depresión por endogamia en la etapa más temprana de crecimiento. Complementariamente, se entrega información poblacional descriptiva acerca de la fenología, atributos florales y densidad poblacional, factores posiblemente asociados con el sistema de reproducción.

\section{MATERIALES Y MÉTODOS}

\section{Sitio y especie de estudio}

Convolvulus chilensis Pers. (Convolvulaceae) es una especie endémica de Chile cuya distribución latitudinal según O’Donell (1957) se extiende desde la II hasta la V Región. Sin embargo, existen colectas de ejemplares entre la II y la VI Región (24는'-34º37' S; herbario CONC: Departamento de Botánica, Universidad de Concepción). Solamente una colecta se ha realizado fuera de este rango (en la VIII Región) y corresponde al tipo (herbario CONC). Esta especie es perenne, herbácea y crece tendida en el suelo o raramente voluble. Presenta una raíz fusiforme vertical, tallos ramificados (los principales dispuestos radialmente) que alcanzan hasta 2,4 m de largo. Las hojas son lanceoladas tomentosas y presentan base cordada con aurículas divergentes. Poseen gran polimorfismo en su forma, tamaño y en la densidad del tomento. La flor es hermafrodita pentámera, posee corola fusionada (diámetro: 2 a $3 \mathrm{~cm}$ ) de color rosado con estambres unidos en su base.
Cada estambre posee dos tecas que se abren longitudinalmente para liberar el polen. El ovario, de forma ovoide, es súpero y bicarpelar. El estigma es bipartido en su extremo (O'Donell 1957). El fruto contiene una a cuatro semillas.

El sitio de estudio se ubica en Aucó (31292' S, 710ㅇ' O, 600-700 m de altitud), dentro de la Reserva Nacional "Las Chinchillas", distante $17 \mathrm{~km}$ de la ciudad de Illapel, IV Región de Chile. El clima se clasifica como semiárido, con precipitaciones esporádicas concentradas en los meses de invierno (junio-agosto). El promedio anual de agua caída es de $170 \mathrm{~mm}$, sin embargo se ha registrado aumentos de hasta $300 \%$ respecto del promedio en los años asociados al fenómeno "El Niño" (Jaksic 2001).

\section{Historia natural}

La población de estudio se encuentra ubicada en una ladera de exposición Norte, formando parte de una comunidad dominada por las especies Echinopsis chilensis, Eulychnia acida y Opuntia ovata (Cactaceae), Flourensia thurifera (Asteraceae), Bridgesia incisifolia (Sapindaceae) y Puya berteroniana (Bromeliaceae). La densidad poblacional de $C$. chilensis no supera los 11,48 individuos $\mathrm{ha}^{-1}$, sin embargo la población reproductivamente activa en la temporada 2003 (individuos que florecieron) fue de 5,18 individuos $\mathrm{ha}^{-1}$ (Gianoli et al., datos no publicados). En un área de 18,66 ha, los individuos de C. chilensis se distribuyen de manera agregada. Esto se determinó mediante el cálculo del SMI (Índice de Morisita estandarizado) según se describe en (Krebs 1999). Así, los valores de SMI en cinco parcelas de área creciente (desde 36 hasta 9.216 $\mathrm{m}^{2}$ ) variaron entre 0,51 y 0,58 ; indicando un patrón espacial agregado (Krebs 1999).

La especie crece principalmente apoyada sobre arbustos, no presenta reproducción clonal y se encuentra sometida a altos niveles de herbivoría: $92,31 \%$ de individuos con daño y 58,93 \pm $4,99 \%$ de tallos ramoneados con respecto al total de tallos del individuo (Gianoli et al. datos no publicados). El daño es provocado por pequeños mamíferos como Lepus capensis (liebre), Oryctolagus cuniculus (conejo) y probablemente por Octodon degus (ratón cola de pincel).

Los visitantes de las flores y potenciales polinizadores de $C$. chilensis se pueden ordenar por tamaño en tres grupos: (1) Insectos de tamaño pequeño (longitud desde la cabeza al ab- 
domen: 1,96-4,33 mm), pertenecientes a las familias Bruchidae, Buprestidae y Melyridae, orden Coleoptera; (2) insectos de tamaño mediano (longitud: 5,02-12,35 mm), pertenecientes a las familias Apoidea, Halictidae y Megachilidae, orden Hymenoptera; (3) insectos grandes $(12,89-19,16 \mathrm{~mm})$, pertenecientes a los géneros Tatochila (Lepidoptera: Pieridae) y Lasia (Diptera: Acroceridae). Esta información se obtuvo durante la ejecución de este estudio (período de máxima floración en la población), capturando entre uno y seis ejemplares de cada uno de los visitantes registrados para su posterior identificación (E. Gianoli, resultados no publicados).

\section{Experimento de polinización controlada}

Para evaluar el sistema de reproducción de $C$. chilensis, en el mes de octubre de 2003, durante el período de máxima floración en la población, se montó un experimento de polinización controlada incorporando los siguientes tratamientos: (1) polinización cruzada manual (PC): Se transfirió polen externo de varios individuos (5 a 10) al estigma de flores previamente aisladas con bolsas de tul en el estado de yema y emasculadas inmediatamente después de la apertura floral, antes de la dehiscencia de las anteras (número de individuos $(\mathrm{NI})=10$; número de flores $(\mathrm{NF})=65) ;(2)$ autopolinización manual (AM): se polinizó flores (previamente aisladas) con polen de la misma flor y de otras flores del mismo individuo ( $\mathrm{NI}=9$; $\mathrm{NF}=58$ ); (3) autopolinización automática (AA): se aisló yemas florales sin ninguna intervención posterior $(\mathrm{NI}=$ 15; NF = 72); (4) apomixis (AP): se aisló y emasculó yemas florales. No se realizó ninguna intervención posterior $(\mathrm{NI}=11 ; \mathrm{NF}=44) ; \mathrm{y}(5)$ polinización natural $(\mathrm{PN})$ (tratamiento control): no se realizó ningún tipo de intervención $(\mathrm{NI}=$ $10 ; \mathrm{NF}=74$ ).

En todos los casos el aislamiento se logró mediante la utilización de bolsas de tul especialmente acondicionadas. Los tratamientos fueron aplicados considerando un pool de 313 flores en 15 individuos. Debido a las bajas tasas diarias de floración y a la alta variación de este atributo (Tabla 1), no se pudo incluir todos los tratamientos en todos los individuos. En cada tratamiento se cuantificó el porcentaje de formación de frutos, calculado como función del número de frutos maduros con respecto al total de flores polinizadas, y se contabilizó el número de semillas producidas por flor tratada.

TABLA 1

Estadística descriptiva de los eventos de floración y atributos florales

Descriptive statistics of flowering events and floral traits

\begin{tabular}{|c|c|c|c|c|c|c|}
\hline Evento / atributo & Promedio & $\mathrm{EE}^{*}$ & Mediana & Mínimo & Máximo & $\mathrm{n}$ \\
\hline Apertura floral (hora cronológica) & $8: 45$ & $9,6 \mathrm{~min}$ & $9: 00$ & $6: 30$ & $10: 15$ & 57 \\
\hline Apertura anteras (hora cronológica) & $9: 35$ & $10,8 \mathrm{~min}$ & $9: 40$ & $7: 00$ & $11: 40$ & 51 \\
\hline Dehiscencia anteras (hora cronológica) & $11: 24$ & $12,6 \mathrm{~min}$ & $11: 30$ & 9:00 & $14: 30$ & 48 \\
\hline Cierre floral (hora cronológica) & $13: 59$ & $10,8 \mathrm{~min}$ & $14: 00$ & $11: 30$ & $15: 50$ & 56 \\
\hline Longevidad floral (h) & 5,25 & 0,09 & 5,00 & 3,92 & 7,00 & 56 \\
\hline Longevidad estructuras masculinas (h) & 1,95 & 0,15 & 1,83 & 0,33 & 3,92 & 45 \\
\hline Flores abiertas por individuo (número día ${ }^{-1}$ ) & 2,14 & 0,56 & 1,18 & 0,00 & 18,00 & 17 \\
\hline Longitud ovario (mm) & 2,01 & 0,05 & 2,00 & 1,25 & 2,65 & 39 \\
\hline Diámetro ovario (mm) & 1,22 & 0,03 & 1,24 & 0,90 & 1,60 & 39 \\
\hline Longitud antera (mm) & 13,70 & 0,20 & 13,63 & 11,34 & 17,74 & 39 \\
\hline Longitud pistilo (mm) & 17,23 & 0,30 & 17,22 & 13,10 & 20,67 & 39 \\
\hline Longitud cáliz (mm) & 8,00 & 0,13 & 8,03 & 5,83 & 9,53 & 39 \\
\hline Relación cáliz/corola & 2,03 & 0,05 & 2,05 & 1,05 & 3,08 & 39 \\
\hline Hercogamia $(\mathrm{mm})$ & 3,52 & 0,34 & 3,87 & $-1,67$ & 7,21 & 39 \\
\hline Área corola $\left(\mathrm{cm}^{2}\right)$ & 6,54 & 0,23 & 6,47 & 2,79 & 10,03 & 39 \\
\hline
\end{tabular}

$* \mathrm{EE}=1$ error estándar 
Se consideró algunos aspectos poblacionales posiblemente relacionados con el sistema de reproducción, determinándose: (1) período de floración, el que se determinó mediante monitoreos realizados a partir del mes de agosto hasta el término de la floración; (2) fenología floral, donde se observó 57 flores $(\mathrm{NI}=8)$ durante un período de siete días con una periodicidad de $30 \mathrm{~min}$ entre las 06:00 y las 22:00 h. Se registró la hora en que ocurrieron los eventos de apertura floral, apertura y dehiscencia de anteras, y cierre floral; (3) morfología floral (Fig. 1) para lo cual se recolectó 40 flores $(\mathrm{NI}=16)$ y se midió los atributos diámetro y longitud de ovario, longitud de estambres, pistilo y cáliz. El área de corola (AC) se estimó en función del área de un pétalo (AP), de manera que $\mathrm{AC}=$ 5*AP. Adicionalmente se calculó la distancia espacial entre sexos, hercogamia (Fig. 1).

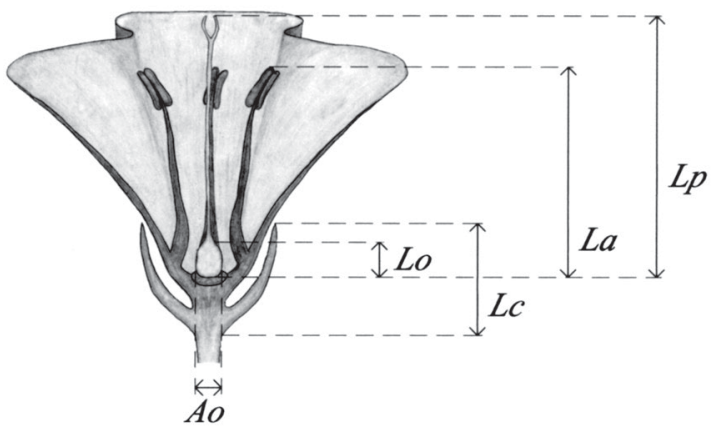

Fig. 1: Corte longitudinal de una flor de Convolvulus chilensis. Atributos florales medidos: $\mathrm{Lp}=$ longitud de pistilo; $\mathrm{La}=$ longitud de estambres; Lc = longitud de cáliz; Lo = longitud de ovario; Ao = diámetro de ovario. Hercogamia $=$ Lp-La

Longitudinal section of a Convolvulus chilensis flower. Measured floral traits: $\mathrm{Lp}=$ pistil length; $\mathrm{La}=$ stamens length; Lc = calyx length; Lo = ovary length; Ao = ovary diameter. Herkogamy $=$ Lp-La.

Efecto del tipo de polinización (autopolinización o polinización cruzada) sobre la progenie

Con el fin de evaluar el efecto del tipo de polinización sobre la progenie, se comparó atributos de semillas y plántulas obtenidas en los tratamientos AM y PC. Las plántulas fueron criadas en condiciones de invernadero $(\mathrm{T}$ máxima promedio $=24{ }^{\circ} \mathrm{C}$; T mínima promedio $=10^{\circ} \mathrm{C} ;$ Fotoperíodo $=14 \mathrm{~h}$ día $/ 10 \mathrm{~h}$ noche; condición lumínica promedio entre las 12:00 y las $16: 00 \mathrm{~h}=700 \mu \mathrm{mol} \mathrm{m}{ }^{-2} \mathrm{~s}^{-1}$ PAR; riego a capacidad de campo). Los atributos comparados fueron: peso de semillas $(\mathrm{n}=$ 317; semillas AM [ $\mathrm{n}=154]$ : ocho familias maternas representadas por 33, 27, 52, 13, $10,9,7$ y 3 individuos; semillas PC $[\mathrm{n}=$ 163]: nueve familias maternas, representadas por $31,31,60,10,10,8,7,4$ y 2 individuos), germinación ( $n=93$; semillas AM $[n=63]$ : cuatro familias maternas representadas por $16,15,16$ y 16 individuos; semillas PC $[\mathrm{n}=$ 30]: cuatro familias maternas, representadas por ocho individuos cada una) y tamaño de plántulas en invernadero ocho semanas después de la germinación en términos de altura total alcanzada (longitud de tallo principal) y número de hojas $(\mathrm{n}=50$; plántulas $\mathrm{AM}[\mathrm{n}=$ 30]: cuatro familias maternas representadas por nueve, siete, siete y siete individuos; plántulas PC $[\mathrm{n}=20]$ : Las mismas cuatro familias maternas, representadas por tres, seis, cinco, y seis individuos). Las semillas colectadas fueron puestas a germinar bajo condiciones de oscuridad y temperatura ambiental ( $\mathrm{T}$ máxima promedio $=24{ }^{\circ} \mathrm{C}$; $\mathrm{T}$ mínima promedio $=10^{\circ} \mathrm{C}$ ) inmediatamente después de haber sido sometidas a escarificación ácida (ácido sulfúrico $98 \%$ por $30 \mathrm{~min}$ ), evaluándose la germinación 21 días después.

\section{Análisis de los datos}

Se determinó el índice de autocompatibilidad (ISI, Ruiz \& Arroyo 1978) como:

$$
\mathrm{ISI}=\frac{N \bar{S} A M}{N \bar{S} P C}
$$

y el índice de autogamia (IAS, Ruiz \& Arroyo 1978) como:

$\mathrm{IAS}=\frac{\% P F_{A A}}{\% P F_{A M}}$

donde $N \bar{s}=$ número de semillas por fruto promedio para el tratamiento indicado como subíndice y $\% P F=$ porcentaje de formación de frutos. Valores de ISI iguales a 1 indican autocompatibilidad; Valores entre 0,2 y 1 indican autocompatibilidad incompleta y valores menores a 0,2 indican autoincompatibilidad. Valores de IAS iguales a 1 indican autogamia completa mientras que valores entre 0 y 1 indican autogamia parcial (Ruiz \& Arroyo 1978).

Para la evaluación entre tratamientos de polinización de diferencias en el porcentaje 
de formación de frutos y el número de semillas por flor tratada se utilizó la prueba de KruskalWallis. Cuando fue necesario se utilizó la prueba a posteriori de Nemenyi, modificada según Dunn (1964; en ZAR 1996: 226-227). El efecto del tipo de polinización (propia o cruzada) sobre los atributos de la progenie se evaluó mediante pruebas $t$ de Student (variables respuesta: peso de semilla, altura total y número de hojas en plántulas) y mediante regresión logística (variable respuesta dicotómica: estado de germinación 1/0).

\section{RESULTADOS}

\section{Sistema de reproducción}

Convolvus chilensis es una especie autocompatible (ISI $=0,96)$ y parcialmente autógama (IAS $=0,81$ ); Posee un sistema de reproducción mixto, en que todos los tratamientos produjeron frutos y semillas maduras. El porcentaje de formación de frutos muestra diferencias entre tratamientos (prueba de Kruskal-Wallis, $\mathrm{H}_{4}=$ 33,79; $\mathrm{P}<0,0001)$. Los tratamientos AA, AM, $\mathrm{PC}$ y $\mathrm{PN}$ no presentaron diferencias entre sí, mientras que el tratamiento de apomixis presentó el menor porcentaje de formación de frutos (Fig. 2). De manera similar, el número de semillas por flor tratada mostró diferencias entre tratamientos (prueba de Kruskal-Wallis, $\mathrm{H}_{4}$ $=64,08 ; \mathrm{P}<0,0001)$, siendo la apomixis la que presenta menor número de semillas en comparación a los otros cuatro tratamientos, los cuales no son distintos entre sí (Fig. 2).

La especie presenta floración extensiva, cuya duración alcanza 22 semanas, iniciándose en el mes de agosto y concluyendo en enero. Algunos individuos aislados permanecen vivos sin perder la biomasa aérea durante la estación seca, floreciendo esporádicamente durante todo el año. La máxima floración ocurre entre los meses de septiembre y octubre, época en la cual los individuos florecen a tasas entre una y 18 flores día ${ }^{-1}$. Las flores se abren solo una vez, un solo día, durante $5,25 \mathrm{~h}$ en promedio, entre las 06:30 y las 15:50 h (Tabla 1). La disponibilidad de polen ocurre durante un período promedio de 1,5 h; entre las 07:00 y las 14:30 h (Tabla 1). Los valores de hercogamia, calculada como la diferencia entre la longitud del pistilo y la longitud de la antera más alta (Fig. 1), mostra- ron una distribución normal (prueba de Kolmorogov-Smirnov, $\mathrm{d}=0,08 ; \mathrm{P}>0,20)$, con un promedio de $3,52 \mathrm{~mm}$ (rango entre $-1,67$ y 7,21 mm) (Tabla 1).

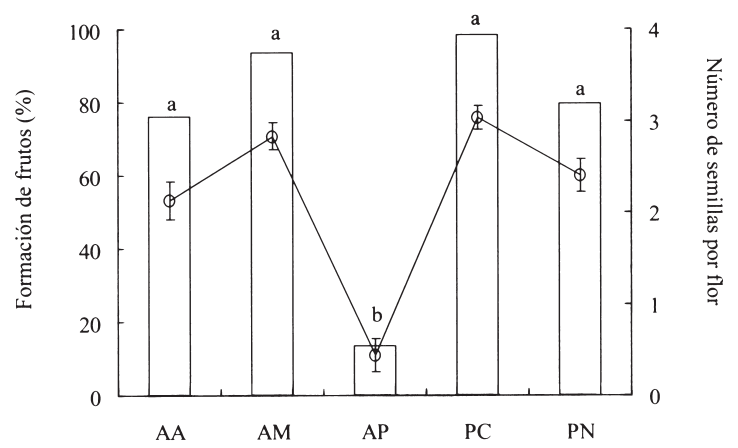

Tratamiento

Fig. 2: Efecto de los tratamientos de polinización $(\mathrm{AA}=$ autopolinización automática, $\mathrm{AM}$ = autopolinización manual, $\mathrm{AP}=$ apomixis, $\mathrm{PC}=$ polinización cruzada, $\mathrm{PN}=$ polinización natural) sobre la formación de frutos, calculada como porcentaje de frutos maduros con respecto al total de flores polinizadas (barras), y sobre el número de semillas por flor tratada (puntos). Letras distintas indican diferencias estadísticamente significativas $(\mathrm{P}<0,05$ en ambos ejes y: formación de frutos y número de semillas por flor tratada) según la prueba a posteriori de Nemenyi. Barras de error indican $1 \mathrm{EE}$.

Effect of pollination treatments $(\mathrm{AA}=$ automatic selfpollination, $\mathrm{AM}=$ manual self-pollination, $\mathrm{AP}=$ apomixis, $\mathrm{PC}=$ cross-pollination, $\mathrm{PN}=$ natural pollination) on fruit formation, calculated as mature fruit percentage with regard to the total pollinated flowers (bars), and on the number of seeds per treated flower (points). Different letters indicate statistically significant differences $(\mathrm{P}<$ 0.05 in both y axis: fruit formation and number of seed per treated flower) according to Nemenyi a posteriori test. Error bars indicate $1 \mathrm{SE}$.

\section{Efectos del tipo de polinización en la progenie}

Al comparar los atributos de la progenie de plantas autopolinizadas con aquellas provenientes de polinización cruzada, se encontró que tanto el peso de las semillas (Tabla 2) como su germinación (Regresión Logística; Wald $=0,2772 ; \mathrm{gl}=1 ; \mathrm{P}>0,05)$ no son distintos entre grupos. Con respecto al tamaño de plántulas, tanto el número de hojas como la altura total no presentaron diferencias entre los grupos (Tabla 2). 
TABLA 2

Efecto del tipo de polinización $(\mathrm{AM}=$ tratamiento de autopolinización manual; $\mathrm{PC}=$ tratamiento de polinización cruzada) sobre atributos de semillas y plántulas

Effect of the type of pollination ( $\mathrm{AM}=$ manual self-pollination treatment; $\mathrm{PC}=$ manual cross-pollination treatment $)$ on seeds and seedlings traits

\begin{tabular}{|c|c|c|c|c|}
\hline \multirow[t]{2}{*}{ Atributo } & \multicolumn{2}{|c|}{ Promedio $\pm \mathrm{EE}$} & \multirow[t]{2}{*}{ Valor de $t$} & \multirow[t]{2}{*}{ Grados de libertad } \\
\hline & $\mathrm{AM}$ & $\mathrm{PC}$ & & \\
\hline Peso de semilla (mg) & $18,9 \pm 0,21$ & $18,2 \pm 0,23$ & $1,93^{N S}$ & 315 \\
\hline Germinación $(\%)^{\mathrm{a}}$ & $93,75 \pm 2,55$ & $96,87 \pm 3,12$ & - & - \\
\hline Número de hojas & $7,8 \pm 0,23$ & $7,3 \pm 0,27$ & $1,38^{N S}$ & 48 \\
\hline Longitud total de tallo $(\mathrm{cm})$ & $40,86 \pm 10,61$ & $30,72 \pm 1,12$ & $0,77^{N S}$ & 48 \\
\hline
\end{tabular}

$N S=\mathrm{P}>0,05 ;$ prueba t de Student;

a $=$ porcentajes de germinación por grupo. Información descriptiva no sujeta a prueba estadística

\section{DISCUSIÓN}

\section{Sistema de reproducción}

La especie perenne $C$. chilensis presentó un sistema de reproducción mixto caracterizado por un alto nivel de autocompatibilidad (ISI = 0,96), lo que es poco frecuente en las especies perennes de la familia Convolvulaceae (21\% de los casos estudiados). Además, la especie mostró una particular facilidad para producir descendencia, la que se expresa en los altos porcentajes de formación de frutos con semillas maduras, exceptuando la apomixis, que presentó una baja formación de frutos (Fig. 2). El tratamiento de apomixis parece poco habitual, pues se presentó solo en un individuo de un total de once evaluados. La ausencia de diferencias en el número de semillas por flor en los tratamientos de AA, AM, PC y PN confirma que $C$. chilensis produce descendencia independientemente del origen del polen. La especie presentó un 93,5\% de formación de frutos a partir de autopolinización manual y 98,6 \% en polinización cruzada. Estos valores, en comparación al 79,7 \% obtenido en el tratamiento control, sugieren que la transmisión del polen no es del todo eficiente, lo que puede deberse a baja disponibilidad y/o eficiencia de polinizadores. El porcentaje de formación de frutos por autogamia (tratamiento de autopolinización automática), sugiere que la especie no depende de polinizadores para asegurar su reproducción. Por otra parte, el alto índice de autogamia (IAS
$=0,81)$ sugiere que la autopolinización en condiciones naturales podría originarse mayoritariamente a nivel intraflor.

El sistema mixto de reproducción en $C$. chilensis, caracterizado por un alto nivel de autocompatibilidad, puede ser ventajoso para la permanencia de esta población. Esto se explicaría considerando que, si bien la polinización cruzada puede representar la mejor opción para la reproducción (mantenimiento o aumento de la diversidad genética: Hamrick \& Godt 1996, Raven et al. 1999), la posibilidad de autopolinización puede resultar favorable en términos de asegurar la reproducción aun considerando que los donantes de polen coespecíficos resulten escasos, tal como ha sido planteado en el contexto de la "reproductive assurance hypothesis" (Stebbins 1950, Morgan et al. 1997).

\section{Factores que podrían asociarse con el sistema de reproducción}

En general, la tasa de exocruza de un genotipo dado resulta de la interacción de atributos determinados genéticamente con factores ecológicos como abundancia de polinizadores y densidad poblacional (Schemske \& Lande 1985). En la población de estudio, diversos factores pueden haber afectado o estar afectando el tipo de reproducción de $C$. chilensis. Antecedentes de historia natural como los altos niveles de daño por herbivoría, o demográficos como la baja densidad poblacional, pueden afectar negativamente la interacción planta-polinizador y el 
éxito reproductivo de las plantas (Strauss 1997, Mustajärvi et al. 2001). Otros factores intrínsecos a la planta como la extensión del período de floración, longevidad floral, tasa diaria de apertura floral y hercogamia pueden considerarse como causa o consecuencia del tipo de sistema reproductivo.

(1) Herbivoría. Algunos autores han encontrado que la herbivoría de tejidos vegetativos puede inducir modificaciones en la arquitectura de la planta y en algunos atributos florales (tiempo a la floración, tamaño/biomasa floral, producción y calidad de néctar) relacionados con el atractivo para polinizadores (Strauss 1997, Juenger \& Bergelson 2000, Elle \& Hare 2002, Strauss \& Murch 2004). La modificación de la arquitectura de la planta y la pérdida de dominancia apical pueden retrasar la floración, pudiendo perder el máximo natural de polinizadores (Juenger \& Bergelson 1997). Por otra parte, se ha reportado que un menor atractivo de los atributos florales puede derivarse de la herbivoría extrafloral y disminuir la actividad de los polinizadores (Strauss et al. 1996). La disminución en el número y duración de las visitas de los polinizadores (vía pérdida del máximo de polinizadores o disminución del atractivo floral) puede traer como consecuencia una disminución de la tasa de exocruza. En el caso de $C$. chilensis, los altos niveles de herbivoría registrados para la población $(58,93 \%$ de tallos ramoneados, véase los métodos) puede incentivar un aumento del crecimiento vegetativo a través de la formación de nuevos tallos por pérdida de dominancia apical. El aumento del crecimiento vegetativo podría retrasar el inicio de la floración afectando así la interacción plantapolinizador y en consecuencia el modo de reproducción.

(2) Densidad poblacional. Se ha planteado que el tamaño y la estructura espacial de una población pueden afectar la dispersión efectiva de polen entre individuos mediante la influencia sobre el comportamiento de los polinizadores (Sih \& Baltus 1987). La baja densidad de individuos con capacidad reproductiva en la población de estudio podría dificultar la reproducción cruzada. Esto debido a pérdidas de polen o disminución de visitas por causa de posamientos en otras especies y/o atascamiento de polen de otras especies en el estigma.

(3) Período de floración. La floración extensa en $C$. chilensis, que se inicia a mediados del invierno y termina a mediados del verano, abarcando 22 semanas, puede ser ventajosa porque permite aumentar la probabilidad de visitas de polinizadores (Arroyo et al. 1981).

(4) Longevidad floral. Para la determinación de la longevidad floral son importantes factores fisiológicos, ecológicos y genéticos (Primack 1985). Su combinación puede dar cuenta de la variación de este atributo dentro de un grupo taxonómico. Las familia Convolvulaceae parece tener exclusivamente "flores de un día" (Primack 1985), aun cuando ocupan variados hábitats a lo largo de su distribución mundial. Esto sugiere que este atributo estaría explicado por factores filogenéticos antes que por factores ecológicos. La corta longevidad de las flores en $C$. chilensis y el breve período en que el polen está disponible (Tabla 1) pueden dificultar el intercambio exitoso de polen entre individuos. Por otra parte, menores longevidades involucran un menor costo para la planta en términos de mantención floral, porcentaje de formación de frutos, producción de néctar, respiración y transpiración floral (Primack 1985, Schoen \& Ashman 1995, Ashman \& Schoen 1997). Factores asociados a las relaciones hídricas en la planta pueden resultar especialmente importantes en este caso, pues el tipo de clima semiárido determina que la disponibilidad de agua durante el verano sea limitante y las pérdidas de agua asociadas al evento de floración puedan representar un alto costo para el individuo (Nobel 1977, Galen et al. 1999).

(5) Tasa diaria de floración. Una alta tasa diaria de aperturas florales favorece el despliegue total de un individuo aumentando su atractivo y promoviendo consecuentemente la visita de polinizadores. Floraciones masivas han sido relacionadas con el aumento de autopolinización entre flores del mismo individuo (geitonogamia) debido al comportamiento de los polinizadores (Primack 1985, De Jong et al. 1993). Las bajas tasas diarias de floración registradas en este estudio reducen esta posibilidad.

(6) Hercogamia. La separación espacial entre sexos es considerada como un método de evitar la autopolinización, de manera que la autopolinización intraflor puede ser facilitada cuando los estambres se encuentran muy cerca del estigma (Dafni 1992, Holsinger 2000). Murcia (1990) relaciona negativamente la deposición de polen propio en el estigma con la hercogamia en la convolvulácea autocompati- 
ble Ipomoea trichocarpa, tanto en ausencia como en presencia de polinizadores. El patrón de una correlación negativa entre hercogamia y autopolinización coincide con el reportado por varios autores para diferentes especies (e.g., Gilia achilleifolia: Takebayashi \& Delph 2000; Datura wrightii: Elle \& Hare 2002). Los valores de hercogamia registrados en este estudio (Tabla 1) pueden estar favoreciendo la autopolinización (autogamia y autopolinización mediada) dada su magnitud. Por una parte, los valores negativos podrían contribuir a explicar el alto índice de autogamia (IAS = 0,81 , vía contacto casual del polen con el estigma (gravedad, viento). Por otra parte, insectos de tamaño mayor que la separación espacial entre los sexos en la flor pueden facilitar el contacto del polen propio con el estigma, provocando autopolinización debido a sus movimientos. En la población de estudio, las flores de $C$. chilensis son visitadas por insectos cuyos tamaños varían entre 1,4 a 5,44 (promedio $=3,4$ ) veces el valor promedio de hercogamia registrado (grupos 2 y 3 descritos en la Metodología: Apoidea, Halictidae, Megachilidae, Pieridae y Acroceridae). Ellos pueden inducir autopolinización mediada aun cuando el estigma sobrepase en altura a las anteras, conducta que ha sido observada en terreno. Sin embargo, estos antecedentes deben tomarse con cautela pues no se cuantificó la frecuencia de visitas de cada tipo de polinizador.

Efectos del tipo de polinización (autopolinización o polinización cruzada) en la progenie

La dirección en que evolucionan los sistemas de reproducción depende del nivel de depresión por endogamia (ID) (Schemske \& Lande 1985). La ID es la mayor fuerza que se opone a la evolución de los sistemas de autopolinización (Charlesworth \& Charlesworth 1987). Convolvus chilensis no expresó ID detectable en los atributos considerados, i.e. la calidad de las semillas y el desempeño de la progenie no fue diferente en los tratamientos AM y PC. Este patrón sugiere un beneficio para la población de estudio en tiempo actual, pues cuando no se logre la reproducción cruzada los individuos podrían asegurar la descendencia de una temporada vía autopolinización sin afectar negativamente por ello el desempeño de la progenie. Algunas consideraciones importantes impiden ser concluyentes en cuanto a la ausencia de detección de ID. En primer lugar, la ausencia actual de ID no implica que esta no haya ocurrido antes. Así, la población en estudio puede haber experimentado la eliminación de alelos desventajosos vinculados a la expresión de algunos de los atributos evaluados (véase estudios de "purging": eliminación de alelos deletéreos (Byers \& Waller 1999). En segundo lugar, el ambiente de protección que ofrece el invernadero puede enmascarar posibles diferencias. Bajo condiciones naturales, la expresión diferencial de caracteres en respuesta al estrés podría permitir reconocer con mayor facilidad diferencias en vigor entre individuos, pues individuos endogámicos pueden ser menos tolerantes al estrés ambiental (Bijlsma et al. 1999, Cheptou et al. 2000). Por último, aquí hemos evaluado atributos en un estado muy temprano de las plántulas. Es posible que se expresen diferencias en la etapa adulta en otros componentes de la adecuación biológica, por ejemplo inicio reproductivo, producción de polen, producción de semillas u otro.

Convolvulus chilensis forma parte del grupo minoritario de especies perennes que presentan autocompatibilidad en la familia Convolvulaceae. El conocimiento de los niveles de endogamia y su efecto sobre las progenies en poblaciones naturales puede contribuir a dilucidar aspectos relacionados con la evolución de sistemas mixtos de reproducción, particularmente la autopolinización. Futuros trabajos podrían dirigirse a evaluar cómo las condiciones ambientales locales, los atributos demográficos y rasgos de historia de vida pueden incidir en el sistema de reproducción, particularmente en las tasas naturales de exocruzas y nivel de endogamia en distintas poblaciones, detectando la variación intraespecífica y sus posibles causas.

\section{AGRADECIMIENTOS}

Agradecemos a Nora Peña, Carmen G. Ossa y Eliser Pardo por su apoyo en las actividades de terreno, y a Eduardo Fuentes-Contreras y Claudio C. Ramírez por la identificación de los insectos. Agradecemos igualmente a CONAF IV Región, especialmente al equipo de guardaparques de la Reserva Nacional "Las Chinchillas" por su excelente disposición al prestar todas las facilidades logísticas necesarias durante el desarrollo de los experimentos en terreno. A Mar- 
cia González-Teuber por el apoyo logístico prestado durante experimento en invernadero. Lohengrin A. Cavieres contribuyó a mejorar significativamente el manuscrito con sus comentarios. Igualmente agradecemos a dos revisores anónimos quienes contribuyeron a mejorar sustancialmente versiones anteriores de este manuscrito. Este trabajo fue financiado por el proyecto FONDECYT 1030702.

\section{LITERATURA CITADA}

AARSSEN LW (2000) Why are most selfers annuals? A new hypothesis for the fitness benefit to selfing. Oikos 89: 606-612.

ARROYO MTK, JJ ARMESTO \& C VILLAGRÁN (1981) Plant phenological patterns in the high andean cordillera of central Chile. Journal of Ecology 69: 205223.

ARROYO MTK \& P USLAR (1993) Breeding system in a temperate mediterrean-type climate montane sclerophyllous forest in central Chile. Botanical Journal of the Linnean Society 111: 83-112.

ARROYO MTK \& F SQUEO (1990) Relationship between plant breeding systems and pollination. En: Kawano $\mathrm{S}$ (ed) Biological approaches and evolutionary trends in plants: 205-227. Academic Press, London, United Kingdom. 417 pp.

ASHMAN T-L \& DJ SCHOEN (1994) How long should flowers live?. Nature 371: 788-791.

ASHMAN T-L \& DJ SCHOEN (1997) The cost of floral longevity in Clarkia temblorensis: an experimental investigation. Evolutionary Ecology 11: 289-300.

BARRETT SC \& CG ECKERT (1990) Variation and evolution of mating systems in seed plants. En: Kawano $S$ (ed) Biological approaches and evolutionary trends in plants: 230-254. Academic Press, London, United Kingdom. 417 pp.

BARRETT SC, LD HARDER \& AC WORLEY (1996) The comparative biology of pollination and mating in flowering plants. Philosophical Transactions of the Royal Society of London B 351: 1271-1280.

BARRETT SCH \& LD HARDER (1996) Ecology and evolution of plant mating. Trends in Ecology \& Evolution 11: 73-79.

BIJLSMA R, J BUNSGAARD \& WF VAN PUTTEN (1999) Environmental dependence of inbreeding depression and purging in Drosophila melanogaster. Journal of Evolutionary Biology 12: 1225-1137.

BYERS DL \& DM WALLER (1999) Do plant populations purge their genetic load? Effects of population size and mating history on inbreeding depression. Annual Review of Ecology and Systematics 33: 479-513.

CHANG S \& MD RAUSHER (1998) Frequency dependent pollen discounting contributes to maintenance of mixed mating system in the common glory Ipomoea purpurea. American Naturalist 152: 671-683.

CHARLESWORTH D \& B CHARLESWORTH (1987) Inbreeding depression and its evolutionary consequences. Annual Review of Ecology and Systematics 18: 237-268.

CHEPTOU PO, E IMBERT, J LEPART \& J ESCARRE (2000) Effect of competition on lifetime estimates of inbreeding depression in outcrossing plant
Crepis sancta (Asteraceae). Journal of Evolutionary Biology 13: 522-553.

CRONQUIST A (1981) An integrated system of classification of flowering plants. Columbia University Press, New York, New York, USA. 1261 pp.

DAFNI A (1992) Pollination ecology. A practical approach. Oxford University Press, Oxford, United Kingdom. 250 pp.

DE JONG TJ, NM WASER \& GL KLINKHAMER (1993) Geitonogamy: the neglected side of selfing. Trends in Ecology \& Evolution 8: 321-325.

DEVALL MS \& LB THIEN (1992) Self-incompatibility in Ipomoea pes-caprae (Convolvulaceae) American Midland Naturalist 128: 22-29.

ELLE E \& D HARE (2002) Environmentally induced variation in floral traits affects the mating system in Datura wrightii. Functional Ecology 16: 79-88.

GALEN C, RA SHERRY \& AB CARROLL (1999) Are flowers physiological sinks or faucet? Cost and correlated of water use by flowers of Polemonium viscosum. Oecologia 118: 461-470.

GALETTO L, A FIONI \& A CALVIÑ̃ (2002) Éxito reproductivo y calidad de frutos en poblaciones del extremo sur de la distribución de Ipomoea purpurea (Convolvulaceae). Darwiniana 40: 25-32.

HAMRICK JL, JW GODT (1996) Effects of life history traits on genetic diversity in plant species. Philosophical Transactions of the Royal Society of London B 351: 1291-1298.

HOLSINGER KE (2000) Reproductive systems and evolution in vascular pants. Proceedings of the National Academy Sciences USA 97: 7037-7042.

HUSBAND BC \& DW SCHEMSKE (1996) Evolution of the magnitude and timing of inbreeding depression in plants. Evolution 50: 54-70.

JAKSIC FM (2001) Ecological effects of El Niño in terrestrial ecosystems of western South America. Ecography 24: 241-250.

JUENGER T \& J BERGELSON (1997) Pollen and resource limitation of compensation to herbivory in Scarlett Gilia, Ipomopsis aggregata. Ecology 78: 1684-1695.

JUENGER T \& J BERGELSON (2000) Does early season browsing influence the effect of self-pollination in Scarlet Gilia? Ecology 81: 41-48.

KREBS CJ (1999) Ecological methodology. Second edition. Addison Wesley, Longman, Inc., Menlo Park, California, USA. $620 \mathrm{pp}$

LLOYD DG (1979) Some reproductive factors affecting the selection of self-fertilization in plants. American Naturalist 113: 67-79.

LLOYD DG (1992) Self- and cross-fertilization in plants. II. The selection of self-fertilization. International Journal of Plant Science 153: 370 - 380.

LOVETT DOUST J \& L LOVETT DOUST (1988) Plant reproductive ecology. Patterns and strategies. Oxford University Press, New York, New York, USA. 344 pp.

MARTIN FW (1970) Self- and interespecific incompatibility in the Convolvulaceae. Botanical Gazette 131: 139-144.

MORGAN MT, DJ SCHOEN \& TM BATAILLON (1997) The evolution of self-fertilization in perennials. American Naturalist 150: 618-368.

MURCIA C (1990) Effect of floral morphology and temperature on pollen receipt and removal in Ipomoea trichocarpa. Ecology 71: 1098-1109.

MUSTAJÄRVI K, P SIIKAMÄKI, S RYTKÖNEN \& A LAMMI (2001) Consequences of plant population size and density for plant-pollinator interactions and plant performance. Journal of Ecology 89: 80-87. 
NOBEL PS (1977) Water relations of flowering in Agave deserti. Botanical Gazette 138: 1-6.

O’DONELL CA (1957) Convolvuloideas chilenas. Boletín de la Sociedad Argentina de Botánica 6: 144-184.

PRIMACK RB (1985) Longevity of individual flowers. Annual Review of Ecology and Systematics 16: 1537.

RAVEN PH, RE EVERT, SE EICHHORN (1999) Biology of plants. W.H. Freeman and Company Worth Publishers, New York, New York, USA. 944 pp.

RUIZ T \& MTK ARROYO (1978) Plant reproductive ecology of a secondary deciduous tropical forest in Venezuela. Biotropica 10: 221-230.

SCHEMSKE DW \& R LANDE (1985) The evolution of self-fertilization and inbreeding depression in plants: II: Empirical observations. Evolution 39: 41-52.

SCHOEN DJ \& T-L ASHMAN (1995) The evolution of floral longevity: Resource allocation to maintenances versus construction of repeated parts in modular organisms. Evolution 49: 131-139.

SCHOEN DJ, MT MORGAN \& T BATAILLON (1996) How does self-pollination evolve? Inferences from floral ecology and molecular genetic variation. Philosophical Transactions of the Royal Society of London B 351: 1281-1290.

SCHLISING RA (1970) Sequence and timing of bee foraging in flowers of Ipomoea and Aniseia (Convolvulaceae). Ecology 51: 1061-1067.

SIH A \& M BALTUS (1987) Patch size, pollinator behavior and pollinator limitation in catnip. Ecology 68: 1679-1690.

STEBBINS GL (1950) Variation and evolution in plants. Columbia University Press, New York, New York, USA. $561 \mathrm{pp}$.

STEBBINS GL (1957) Self-fertilization and population variability in the higher plants. American Naturalist 91: 337-354.

STRAUSS SY, JK CONNER \& SL RUSH (1996) Foliar herbivory affects floral characters and plant atractiveness to pollinators: Implications for male and female plant fitness. American Naturalist 147: 1098-1107.

STRAUSS SY (1997) Floral characters link herbivores, pollinators and plant fitness. Ecology 78: 16401645.

STRAUSS SY \& P MURCH (2004) Towards an understanding of the mechanism of tolerance: compensating for herbivore damage by enhancing a mutualism. Ecological Entomology 29: 234-239.

STUCKY JM \& RL BECKMANN (1982) Pollination biology, self-incompatibility, and sterility in Ipomoea pandurata (L.) G.F. W. MEYER (Convolvulaceae). American Journal of Botany 69: 1022-1031.

TAKEBAYASHI N \& LF DELPH (2000) An association between a floral trait and inbreeding depression. Evolution 54: 840-846.

USHIMARU A \& K KIKUZAWA (1999) Variation of breeding system, floral rewards, and reproductive success in clonal Calystegia species (Convolvulaceae). American Journal of Botany 86: 436-446.

WEAVER SE \& WR RILEY (1982) The biology of canadian weeds, 53. Convolvulus arvensis L. Canadian Journal of Plant Science 62: 461-472.

WILLMONTT AP \& A BÚRQUEZ (1996) The pollination of Merremia palmeri (Convolvulaceae): Can hawk moths be trusted? American Journal of Botany 83: 1050-1056.

ZAR JH (1996) Biostatistical Analysis. Prentice Hall, Upper Saddle River, New Jersey, USA. 660 pp. 